\section{Keeping the Sun in proportion}

\section{Andrew A. Lacis and Barbara E. Carlson}

GLOBAL warming observed over the past century has focused attention on manmade greenhouse gases, presumed to be the likely cause. Some researchers, however, have expressed doubt, wondering whether the Sun - our ultimate source of energy - might actually be variable (as stars similar to the Sun are found to be), and thus account for the bulk of the observed climate change. The expressed hope is that future warming due to further greenhouse-gas emissions might therefore not be significant. Two papers in the issue ${ }^{1,2}$ put this idea to the test and find it wanting.

The annually averaged global-mean surface air temperature rose by $0.8{ }^{\circ} \mathrm{C}$ between 1880 and 1990 . This change is broadly accepted, despite the inherent problems of incomplete global coverage, changing observation practices and urbanization. Increases in greenhouse gas concentrations, $\mathrm{CO}_{2}, \mathrm{CH}_{4}, \mathrm{NO}_{2}$ and chlorofluorocarbons have been accurately measured over the same period. Their opacities, which account for the $2 \mathrm{~W} \mathrm{~m}^{-2}$ radiative forcing of the climate incurred since 1850 , are also accurately known. Reflecting the accelerating pace of industrialization and population growth, 23 per cent of this climate forcing has been incurred over the past decade alone ${ }^{3}$.

However, the observed decadal and interdecadal variability of the global mean temperature are clear indications that factors other than greenhouse-gas forcing must also have contributed significantly to the observed temperature change. In particular, the sharp rise in mean temperature in the 1920 s and the subsequent decline during 1940-70 are not at all consistent with the steady increase in greenhouse-gas levels (see Fig. 2 of Schlesinger and Ramankutty ${ }^{2}$ on page 332 ). This inability to model the temperature record with greenhouse-gas forcing alone has led some investigators to question model results of the greenhouse effect and to suggest solar variability as an alternative explanation.

\section{Maunder Minimum}

Although investigating the past behaviour of the Sun is somewhat speculative, the period from 1645 to 1715 , known as the Maunder Minimum, was nevertheless particularly striking, in that all sunspot activity ceased and the world experienced simultaneously a cold period referred to as the Little Ice Age. With no other source of readily identifiable climate forcing to explain this unusual period of cold temperatures, some researchers were led to postulate that changes in the solar ouput of the order of 0.5 per cent might provide an explanation. Thus it was suggested ${ }^{4}$ that the Sun may go through cycling and noncycling phases with the noncycling phase of the Maunder Minimum corresponding to reduced solar output. Using $\mathrm{Ca}$ II $\mathrm{H}$ - and $\mathrm{K}$-line emission as an index of the Sun's brightness, Lean et al. ${ }^{5}$ matched and analysed the emissions observed for noncycling stars, finding that the solar irradiance may have decreased below its current mean level by 0.24 per cent during the Maunder Minimum. The decrease in global mean temperature due to this reduction in total solar irradiance is in the range $0.2-0.6{ }^{\circ} \mathrm{C}$, comparable to the estimated $1{ }^{\circ} \mathrm{C}$ decrease associated with the Little Ice Age.

Direct measurement of the total solar irradiance during the past sunspot cycle has revealed variations of the order of only 0.1 per cent. The larger variability of the solar irradiance, operating over timescales of decades and centuries, must necessarily be inferred indirectly by correlating some measure of solar change with the historical temperature record. The more successful studies have shown that changes in the solar umbrapenumbra ratio, sunspot cycle envelope and, more recently, changes in length of the solar-cycle period can be closely correlated with the global temperature trend $^{6}$. Although variations in the umbra-penumbra ratio, sunspot envelope, and length of the solar cycle may serve as a useful proxy for changes in solar magnetic-field strength and convective activity that act to modulate solar irradiance, the lack of a clear physical mechanism is a definite drawback.

Kelly and Wigley have now used an energy-balance model to investigate the effects of greenhouse and solar forcing over the period 1765-1985. Their climate simulations, on page 328 of this issue ${ }^{1}$, provide circumstantial support for a link between solar-cycle length and irradiance, but show the solar contribution to the global-mean temperature change to be much less than that due to increasing greenhouse gases and other anthropogenic effects. Schlesinger and Ramankutty, on page 330 of this issue ${ }^{2}$, use a hemispherically resolved version of their climate-ocean model to investigate the effect of greenhouse gas, aerosol and solar forcing over the period 1638-1985. They also conclude that greenhouse gases will continue to dominate global warming, but note that changes in solar irradiance modify the predictions and, in fact, decrease the predicted temperature change associated with a doubling of atmospheric $\mathrm{CO}_{2}$ by nearly a half.

Kelly and Wigley provide the caveat that the solar-irradiance record is sensitive to the filtering method used to construct the record from the cyclelength data, limiting the utility of this approach until the physical mechanism linking the solar-cycle length to solar irradiance is better understood. An additional caveat is that spectral measurements of the solar irradiance changes during the past sunspot cycle show that the solar ultraviolet radiation takes a disproportionate share of the variability. Wavelengths short of $300 \mathrm{~nm}$ contribute 1 per cent to the total irradiance, but they contribute 20 per cent of the observed variability ${ }^{7}$.

\section{Ultraviolet radiation}

An increase in solar ultraviolet radiation, which is absorbed primarily in the stratosphere, would cause local stratospheric heating with the excess thermal energy being radiated directly to space. This produces no net forcing at the tropopause, hence no increase in surface temperature. Thus, actual solar irradiance variations may be less effective than model results, which assume spectrally uniform changes, imply.

Solar monitoring is clearly needed to establish the long-term variability of solar ultraviolet irradiance. Two solar spectral irradiance experiments (SUSIM and SOLSTICE) on the Upper Atmosphere Research Satellite (UARS) will measure the ultraviolet irradiance within the critical 115-415 $\mathrm{nm}$ spectral range. Along with the active cavity radiometer ACRIM II, also on UARS, there is reason to hope that, for the time being, solar-irradiance will be adequately monitored. But to establish the solarirradiance-climate connection properly will take continuous monitoring over at least several decades. In the meantime, it should be recognized that of the different contributors to climate change, the greenhouse effect of trace-gas forcing is the most accurately documented and the best understood. Policy makers should not be distracted from their vital task of controlling greenhouse-gas emissions.

Andrew A. Lacis and Barbara E. Carlson are at the Goddard Institute for Space Studies, 2880 Broadway, New York. New York 10025, USA.

\footnotetext{
1. Kelly, P. M. \& Wigley, T. M. L. Nature $360,328-330$ (1992).

. Schlesinger, M. E. \& Ramankutty. N. Nature $\mathbf{3 6 0}$ 330-333 (1992).

3. Hansen. J., Lacis, A. \& Prather, M. J. geophys. Res. 94 16417-16421 (1989)

4. Baliunas, S. \& Jastrow, R. Nature 348, 520-523 (1990).

5. Lean, J., Skumanich, A. \& White, O. Geophys. Res. Lett. 19, 1591-1594 (1992)

6. Friis-Christensen, E. \& Lassen, K. Science 254, 698 700 (1991)

7. Lean, J. Science 244, 197-200 (1990)
} 